\title{
COHERENCE BETWEEN FLUCTUATIONS IN BLOOD FLOW AND TISSUE OXYGEN SATURATION
}

\author{
ALAN BERNJAK ${ }^{a}$, ANETA STEFANOVSKA ${ }^{b}$, PETER V.E. McCLINTOCK ${ }^{c}$ \\ Department of Physics \\ Lancaster University, Lancaster, LA1 $4 Y B, U K$ \\ ${ }^{a} a . b e r n j a k @ l a n c a s t e r . a c . u k ;{ }^{b}$ aneta@lancaster.ac.uk; ${ }^{c}$ p.v.e.mcclintock@lancaster.ac.uk \\ P. JANE OWEN-LYNCH \\ Biomedical and Life Sciences Division, School of Health and Medicine \\ Lancaster University, Lancaster, LA1 $4 Y Q$, UK \\ j.owen-lynch@lancaster.ac.uk \\ PETER B.M. CLARKSON \\ Cardiology Department \\ Raigmore Hospital, Old Perth Road, Inverness, IV2 3UJ, UK \\ peter.clarkson1@nhs.net \\ Received (received date) \\ Revised (revised date)
}

\begin{abstract}
We have determined the wavelet phase coherence between simultaneously-recorded microvascular blood flow and oxygen saturation signals from 88 healthy subjects, thus enabling us to study their common fluctuations. Measurements were taken for 30-minutes from the arm and leg, at two depths. In the skin, blood flow and oxygen saturation were found to be coherent both at the cardiac frequency and below $0.1 \mathrm{~Hz}$ down to about 0.01 $\mathrm{Hz}$. Coherence in the arm extends to lower frequencies than that in the leg. From the deeper recordings, no coherence was found on either limb. The existence of coherence between skin blood flow and oxygen saturation demonstrates causal connections between them within certain frequency ranges. The method has yielded the first detailed insight into the dynamics of blood oxygenation.
\end{abstract}

Keywords: Wavelet phase coherence, blood flow, oxygen saturation, microcirculation.

\section{Introduction}

Normal cellular function requires a continuous supply of oxygen. In higher animals a cardiovascular system is used to provide for its convective delivery to the smallest branches of the network of blood vessels - the microcirculation - from which oxygen then passes to the cells through passive diffusion. The oxygen is transported through the cardiovascular system by hemoglobin $(\mathrm{Hb})$, an iron-containing protein in the red blood cells. The Hb oxygen saturation in the blood consists of a steady level that has been extensively studied in terms of time-averages, in both the resting 
state and during exercise. We discuss here oscillatory fluctuations around this level, similar to those that occur in blood flow. In the latter case, studies of the oscillations measured by non-invasive laser-Doppler flowmetry [1-4], have brought much insight into the underlying physiological processes. Oscillations have also been observed in recordings of tissue oxygenation $[5,6]$. Vasomotion might have a beneficial effect on tissue oxygenation, but their relationship is unclear.

In this paper we investigate the interactions between local microvascular blood flow and oxygen saturation by applying the wavelet phase coherence method to simultaneously recorded signals. Techniques for data acquisition are described in Section 2. The wavelet transform and wavelet phase coherence methods are summarised in Section 3, followed in Section 4 by their application to data recorded from 88 healthy subjects. We discuss the results and draw conclusions in Sections 5 and 6 , respectively.

\section{Methods for measurement of skin blood perfusion and oxygenation}

\subsection{Laser-Doppler flowmetry}

Blood flow in the skin was measured by laser-Doppler flowmetry (LDF), based on the technique introduced by Stern [7] and developed by Nilsson et al [8]. Infrared light is delivered to the skin via a flexible optical fiber. The light backscattered from red blood corpuscles is collected, and the distribution of the Doppler shift indicates their average speed and concentration. Strictly, the output value from the LDF device represents flux. However, in the literature flux is often used interchangeably with perfusion or blood flow, assuming a constant concentration of blood corpuscles. The LDF measurements in the present work were made with the O2C ("oxygen to see") device manufactured by LEA Medizintechik, Germany. In addition to blood flow, the device with the specific probe (LF-2 flat probe) enables simultaneous acquisition of $\mathrm{Hb}$ oxygen saturation (see below) in the same location. A near-infrared laser diode $(830 \mathrm{~nm}, 30 \mathrm{~mW})$ is the source of light used to measure blood flow. The light is brought to the skin through optical fibres and the collector fibres are set at two different distances to allow for recordings from two depths. The exact measurement depth depends on tissue properties but the shallow recording (fibre separation $=2 \mathrm{~mm}$ ) reaches to around $1 \mathrm{~mm}$ in the skin (typical for LDF devices) while the deeper recording (fibre separation $=8 \mathrm{~mm}$ ) can reach several $\mathrm{mm}$ deep. In the text we refer to the two depths as "Skin" and "Deep". LDF recordings provide flux expressed in arbitrary perfusion units (AU or PU) on account of the so called "biological zero": even during occlusion, when the blood flow is completely blocked, the instrument yields finite readings due to Brownian motion of the red blood cells and vascular wall movement, as well as movement due to mechanical contraction of the surrounding tissue. 


\subsection{Oxygenation measurements}

Tissue oxygenation depends on the balance between oxygen delivery and utilization [9], but oxygen saturation does not reflect tissue oxygenation directly because the affinity of oxygen to hemoglobin is affected by physiological factors such as e.g. $\mathrm{pH}$ or body temperature. In the current study $\mathrm{Hb}$ oxygen saturation was measured by optical reflectance spectroscopy, a form of near-infrared spectroscopy (NIRS). In general, NIRS enables non-invasive, in vivo, continuous detection of changes in oxygenated haemoglobin, deoxygenated haemoglobin or total haemoglobin (blood volume). It is based on the Beer-Lambert law [10] which relates the absorption of light to the properties of the tissue through which it passes. Oxygenated and deoxygenated hemoglobin have different absorption characteristics in the near-infrared range (700-1000 $\mathrm{nm})$ and the measured absorption can be used to determine the local average oxygen saturation of the hemoglobin. Absolute concentrations are difficult to calculate accurately because the exact geometrical parameters (path of the scattered light) in the tissue are not known. Mean baseline values of oxygenation saturation and responses to provocation have been intensively investigated in the muscles [10] and brain [11]. Recent studies report, analyze and discuss spontaneous fluctuations of the $\mathrm{Hb}$ oxygen saturation in time $[5,6,12,13]$.

In the experiments to be reported below, we measured $\mathrm{Hb}$ oxygen saturation with the $\mathrm{O} 2 \mathrm{C}$ device, using the LP-2 probe. A difference from standard NIRS is that the $\mathrm{O} 2 \mathrm{C}$ is not restricted to specific frequencies. White light (halogen lamp) is shone onto/into the skin and a wide spectrum of back-reflected light (650-795 $\mathrm{nm}$ from the skin and $500-630 \mathrm{~nm}$ from the deeper tissue) is analyzed to estimate oxygen saturation. By using the same probe separations as for the LDF, the sampled areas of tissue for the oxygen saturation and LDF recordings overlap each other. All signals are sampled at $40 \mathrm{~Hz}$, but oxygen saturation data are refreshed once per second. The output values of all data are discretized to integers.

Using the $\mathrm{O} 2 \mathrm{C}$, the signals are recorded from the venous end of the capillaries. Oxygen saturation in these vessels can be much lower than in the large veins, because the venous blood includes "shunt-blood" which does not pass oxygen consuming cells. To determine local oxygen consumption accurately, oxygen saturation should be known on both the arterial and venous sides, together with the hemoglobin levels. The fact that oxygen saturation and blood flow are measured simultaneously in the same volume enables us to investigate the relationship between the two processes and promises to yield improved understanding of the mechanisms of oxygen delivery within the microcirculation.

\section{Wavelet transform and wavelet phase coherence}

The cardiovascular system can be described as a set of at least six coupled oscillators whose characteristic frequencies vary in time $[1,3]$. The associated physiological processes generate complex oscillatory patterns in the blood flow, especially at the level of capillaries. Wavelet analysis with the Morlet mother wavelet can detect 
these oscillations with logarithmic frequency resolution, and follow the variations of their frequencies and amplitudes in time $[1,2]$. By this means, six frequency intervals were defined: I: $0.6-2 \mathrm{~Hz}$ (cardiac activity), II: $0.145-0.6 \mathrm{~Hz}$ (breathing), III: $0.052-0.145 \mathrm{~Hz}$ (myogenic activity), IV: $0.021-0.052 \mathrm{~Hz}$ (neurogenic activity), V: $0.0095-0.021 \mathrm{~Hz}$ (NO-related endothelial) and VI: $0.005-0.0095 \mathrm{~Hz}$ (non-NO endothelial activity) $[3,4]$. The blood flow signals thus reflect the non-autonomous character of the underlying oscillatory processes and we expect similar modulations to be present in the oxygen saturation recordings.

The wavelet transform is a method that provides for the complex transformation of a time series from the time to the time-frequency domain. It involves convolving the time series $g(u)$ with a family of generally non-orthogonal basis functions, generated from the mother wavelet:

$$
g(s, t)=\frac{1}{\sqrt{s}} \int_{-\infty}^{\infty} \psi\left(\frac{u-t}{s}\right) g(u) d u .
$$

In the above expression $\psi$ is the Morlet mother wavelet, scaled by the factor $s$ and translated in time by $t$. The Morlet wavelet is a complex sinusoid modulated by the Gaussian function:

$$
\psi(u)=\frac{1}{\sqrt[4]{\pi}} \cdot e^{-i 2 \pi u} \cdot e^{-u^{2} / 2},
$$

where $i=\sqrt{-1}$. Scaling of the wavelet enables the detection of oscillations with different frequencies, while its translation in time allows one to follow how the spectra evolve in time. The rationale for using the Morlet wavelet is that its Gaussian envelope provides good localization of events in both time and frequency; in time, approximately 6-7 periods are used to detect the power of each oscillatory component. Moreover, there is a straightforward inverse relationship between the scaling factor $s$ and its corresponding frequency: $f=1 / s$.

As noted above, oscillations in the cardiovascular system manifest over the wide frequency range from $0.005 \mathrm{~Hz}$ to $2 \mathrm{~Hz}$ in recordings of around 30 minutes duration. For longer recordings, even lower frequency oscillations would be expected to appear if problems of movement artifacts and non-stationarity could be overcome. To accommodate both high and low frequencies, logarithmic frequency resolution is used. Starting with scale $s=0.5$ (corresponding to the highest frequency $2 \mathrm{~Hz}$ ), the wavelets are stretched by factor 1.05 , until $s=200$ is reached (corresponding to the lowest frequency $0.005 \mathrm{~Hz}$ ). The resolution in time is set to $1 \mathrm{~s}$. The two most important aspects of analyzing cardiovascular signals with wavelets are the adjustable window length, so that each oscillatory component is detected with its corresponding window length, and the logarithmic frequency resolution, which enables accurate characterization of the low frequency oscillations.

\subsection{Wavelet phase coherence}

With the complex Morlet wavelet, the wavelet coefficients are complex numbers in the time-frequency plane. They define the absolute amplitude and instantaneous 
relative phase for each frequency and time, on a grid. The phase information can be used to investigate relationships between the oscillations from different signals. For example, two noisy signals can contain oscillations at the same frequency but it may not be known whether they are causally related. Wavelet phase coherence can uncover possible relationships by evaluating how well the instantaneous phases from the two signals are matched $[14,15]$.

First, instantaneous phases are calculated at each time $t_{n}$ and frequency $f_{k}$ for both signals: $\phi_{1 k, n}$ and $\phi_{2 k, n}$. Their relative phase difference is then obtained as $\Delta \phi_{k, n}=\phi_{2 k, n}-\phi_{1 k, n}$. The sine and cosine components of the phase differences are calculated and averaged in time for the whole length of the signal. Finally, the phase coherence function is defined as:

$$
C_{\phi}\left(f_{k}\right)=\sqrt{\left\langle\cos \Delta \phi_{k, n}\right\rangle^{2}+\left\langle\sin \Delta \phi_{k, n}\right\rangle^{2}} .
$$

The phase coherence function $C_{\phi}\left(f_{k}\right)$ has a value between 0 and 1 . It quantifies the tendency for the phase difference between the two signals at a particular frequency to remain constant (where the phase coherence value would be close to 1). When two oscillations are unrelated, their phase difference changes continuously in time and so their phase coherence is close to zero.

Phase coherence values must, however, be interpreted with care. In finitelength signals, low-frequency components are represented by fewer periods than high-frequency components. Consequently, less variation of phase difference occurs at low-frequencies, resulting in an artificially increased phase coherence. In fact, the phase coherence between two completely unrelated signals increases monotonically towards lower frequencies. To identify a frequency-dependent floor representing truly significant coherence, we employed surrogate analysis [16]. Amplitudeadjusted Fourier transform (AAFT) surrogate signals were generated by shuffling the phases of the original time series to create new time series with the same means, variances, autocorrelation functions (and therefore, the same power spectra) as the

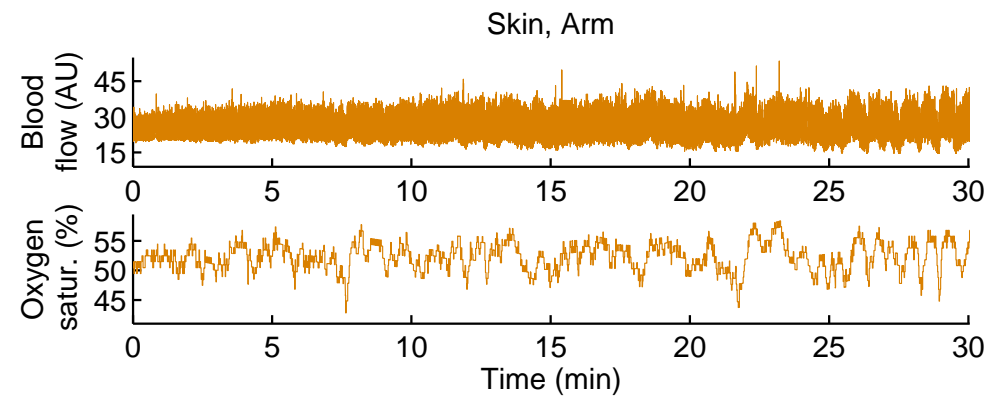

Fig. 1. Simultaneous recordings of blood flow using laser-Doppler flowmetry (top panel), and oxygen saturation using optical reflectance spectroscopy (bottom). The signals were simultaneously recorded with the $\mathrm{O} 2 \mathrm{C}$ instrument on the same location from the skin of the right arm. 
Table 1. Median values and ranges [25th percentile and 75th percentile] of blood flow and oxygen saturation $(\mathrm{N}=88)$.

\begin{tabular}{|c|c|c|c|c|c|c|}
\hline Signal, probe position & & Skin & & & Deep & \\
\hline Blood flow (AU), Arm & 18.85 & {$[11.70$} & $34.81]$ & 77.31 & {$[54.50$} & $128.06]$ \\
\hline Blood flow (AU), Leg & 21.80 & {$[16.62$} & $28.73]$ & 73.44 & {$[49.00$} & $112.36]$ \\
\hline Oxygen saturation (\%), Arm & 46.68 & {$[41.20$} & $55.74]$ & 87.54 & {$[84.82$} & $89.34]^{\prime}$ \\
\hline Oxygen saturation (\%), Leg & 43.35 & 36.35 & $53.70]$ & 75.54 & {$[65.58$} & $80.65]$ \\
\hline
\end{tabular}

original sequences, but without any phase relations. We averaged 100 wavelet phase coherences calculated from surrogate signals, and considered that wavelet coherence from the original recording was statistically significant when it was two standard deviations above the mean surrogate coherence.

\section{Experiments on coherence between blood flow and oxygen saturation}

Using the $\mathrm{O} 2 \mathrm{C}$ with two probes (LF-2), simultaneous measurements of blood flow and oxygen saturation were recorded for 30 minutes from 88 healthy normotensive subjects ( 50 females, 38 males, aged $48 \pm 17$ years, age range $18-90$ years, systolic blood pressure $<150 \mathrm{mmHg}$, diastolic blood pressure $<90 \mathrm{mmHg}$ ), lying relaxed in a supine position. All the signals were sampled at $40 \mathrm{~Hz}$. The probes were positioned on the right arm (forearm, inner side and center) and right leg (middle of shinbone). Skin temperature was measured using a temperature sensor (Thermilinear, YSI Inc., USA) placed on the right forearm and the group skin temperature was $31.8 \pm 1.3{ }^{\circ} \mathrm{C}$. Simultaneous recordings of blood flow and oxygen saturation in the skin are shown in Fig. 1.

Group median values and ranges of blood flow and oxygen saturation are presented in Table 1. Data are given for both depths and locations. For each subject, the average across the whole recording was calculated.

Median wavelet power spectra of blood flow and oxygen saturation signals from the arm are presented for the group in Fig. 2. Spectra of signals recorded at two depths (skin and deeper) are compared in terms of (a, b) absolute and (c, d) normalized power. Normalized spectra were calculated by dividing the power spectra with the total power within the whole frequency range from $0.005 \mathrm{~Hz}$ to $2 \mathrm{~Hz}$ for each subject. A paired signed rank test was used to determine statistically significant differences $(P<0.05)$ between the two depths for each specific frequency (indicated by light grey shading). Average power and normalised power were also calculated within each of the six frequency intervals. Significant differences within the intervals are indicated by asterisks.

Blood flow recorded from the deeper tissue has significantly more power across the whole frequency range compared to blood flow from the skin (Fig. 2a). The comparison of normalised power spectra indicates, that the distribution of power is very similar (Fig. 2c) in both cases. Note that the significant difference around 


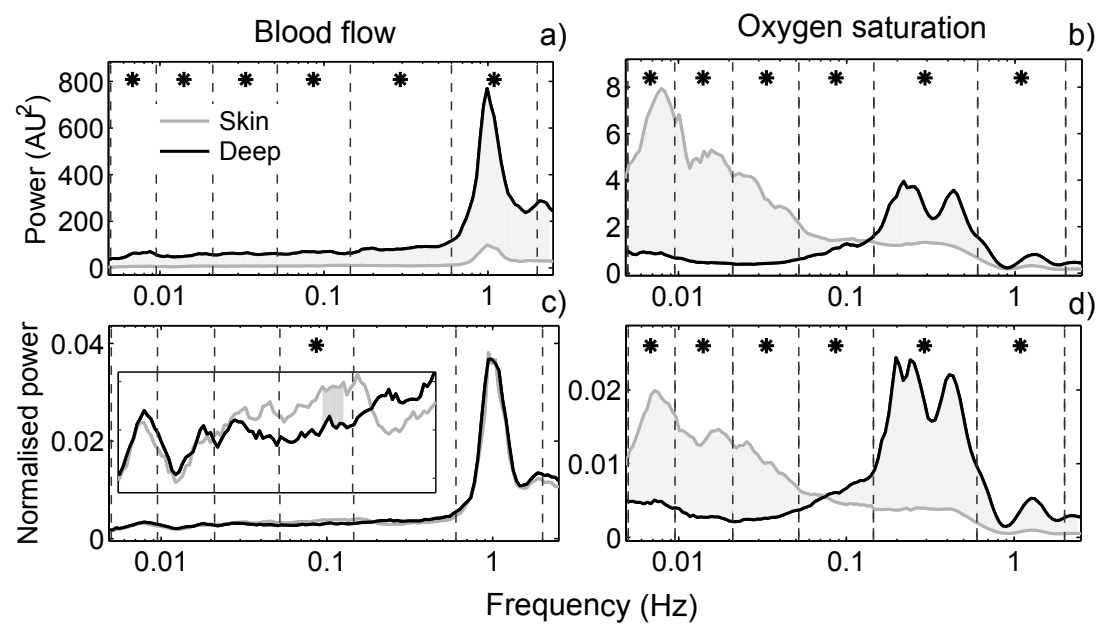

Fig. 2. Wavelet power spectra of signals recorded from the skin and deeper tissue of the arm are compared within the 0.0095-2 Hz frequency range. Group median spectra of blood flow (a,c) and oxygen saturation (b,d) are presented in terms of absolute $(a, b)$ and normalised $(c, d)$ power spectra. A paired test was used to calculate significant differences $(P<0.05)$ at specific frequencies (light grey shading) or within the defined frequency intervals (indicated by asterisks). Blood flow spectra are similar for recordings from the skin and deeper tissue $(\mathrm{a}, \mathrm{c})$, whereas the oxygen saturation spectra are clearly different $(b, d)$. The inset in $(c)$ is to illustrate the low frequency contribution in greater detail.

$0.1 \mathrm{~Hz}$ (asterisk) is obtained by a paired test. In contrast to blood flow, spectra of oxygen saturation from the skin and from the deeper tissue are clearly different. In the deeper tissue, most of the power is concentrated around the respiratory frequency while oxygenation spectra from the skin contain most of the power at frequencies below $0.1 \mathrm{~Hz}$ (Fig. 2b,d). The findings are similar for both arm and leg.

A comparison of skin blood flow power spectra from the arm and leg reveals significantly higher power at frequencies below $1 \mathrm{~Hz}$ for the arm compared to the leg (Fig. 3a). In terms of distribution of power, blood flow in the leg contains less power in the cardiac and the endothelial-related frequency intervals compared to the arm (Fig. 3b). Note that in the current study, LDF recordings were taken from the forearm and on the shin bone in contrast to previous work [1-4], where the probes were positioned on the bony prominences on the wrist and the ankle.

Wavelet phase coherence between blood flow and oxygen saturation was calculated for both arm and leg, at both depths. The median coherence for 88 subjects is presented in Fig. 4. Results from skin on the arm and leg are shown in panels (a) and (b), respectively; results from the deeper measurements on the arm and leg are shown in (c) and (d). For each pair of recordings, 100 AAFT surrogate signals and their phase coherence were calculated. The mean coherence is indicated by the lower, dash-dotted line. The higher dashed line indicates a level two standard deviations above the mean for each frequency. On the arm, there is a statistically 


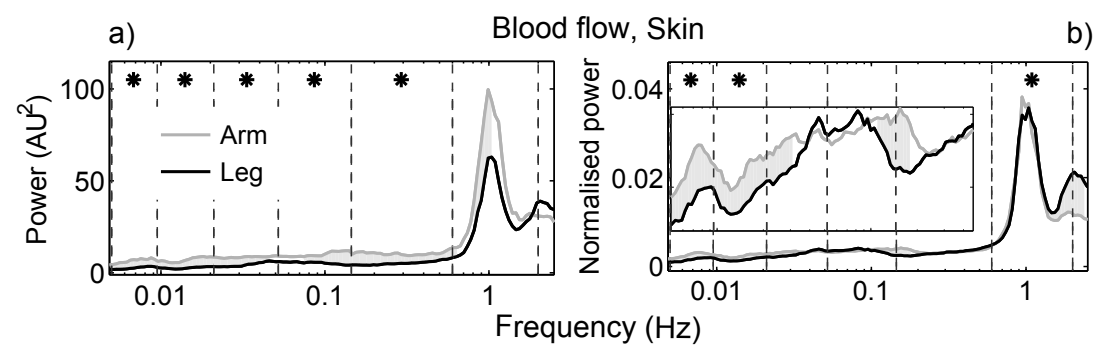

Fig. 3. The wavelet power spectra of skin blood flow signals from the arm and leg are compared within the $0.0095-2 \mathrm{~Hz}$ frequency range. A paired test was used to reveal significant differences $(P<0.05)$ at specific frequencies (grey shading) or within the defined frequency intervals (indicated by asterisks). Median group power is significantly higher in the arm compared to the leg (a), while the normalized spectra show that in terms of proportion of power the differences are significant within the cardiac and endothelial-related intervals (b). The inset provides greater detail of the low frequency components.

significant phase coherence between blood flow and oxygen saturation across a wide frequency range from interval III down to interval VI, and also at the cardiac frequency in interval I (Fig. 4a). On the leg, the coherence is higher at the cardiac frequency compared to the arm, but it is lower or non-significant within intervals $\mathrm{V}$ and VI (Fig. 4b). There is no coherence between blood flow and oxygen saturation recorded from deeper tissue, neither on the arm nor on the leg (Figs. 4c,d).

\section{Discussion}

Coherence between simultaneously measured blood flow and oxygen saturation in the same catchment volume in the microcirculation has not previously been reported. Following a theoretical work [17] suggesting that oscillations in microcirculatory flow can have a significant effect on tissue oxygenation, Goldman \& Popel [18] used their capillary network model to identify the largest effect in the frequency range between $0.025 \mathrm{~Hz}$ and $0.05 \mathrm{~Hz}$. This interval coincides with our interval IV, where we observe significant coherence for skin on both arm and leg. Studies of interactions between simultaneously measured signals are few and mostly involve cerebral circulation. Apart from this, the approaches used to measure flow or oxygenation are often invasive and not focused on the same tissue. To our knowledge, the $\mathrm{O} 2 \mathrm{C}$ is unique in its ability to measure both signals from the same location, non-invasively, and with a high temporal resolution. Using an O2C, Thorn et al. [12] performed measurements similar to ours and identified oscillations in skin oxygen saturation below $0.02 \mathrm{~Hz}$. Based on parameters, defined in the time domain, they associated them with oscillations in skin blood flow on the arm. We show significant coherence at this frequency (interval V) for the arm, but only partly so for the leg. The difference between the limbs in terms of coherence in the skin might be related to the relatively higher contribution of blood flow oscillations at low 


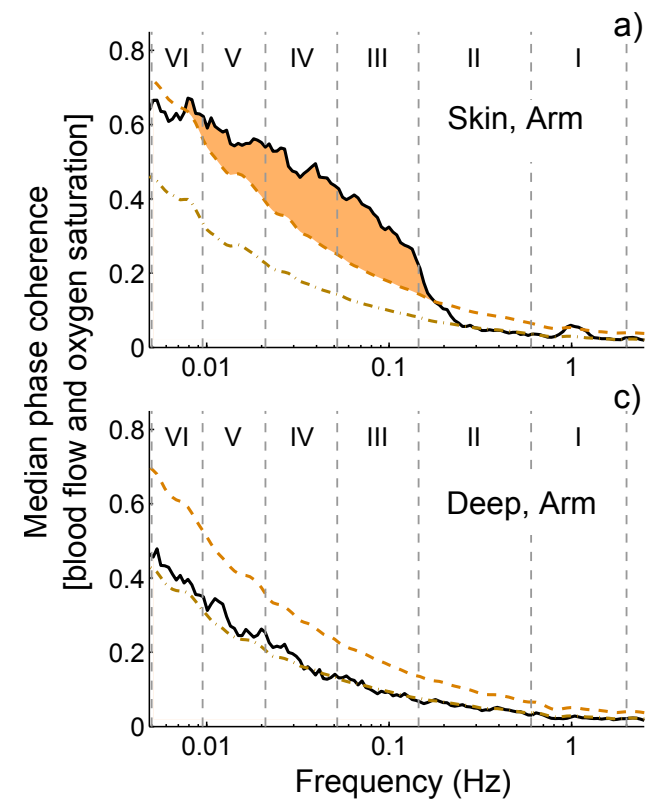

)

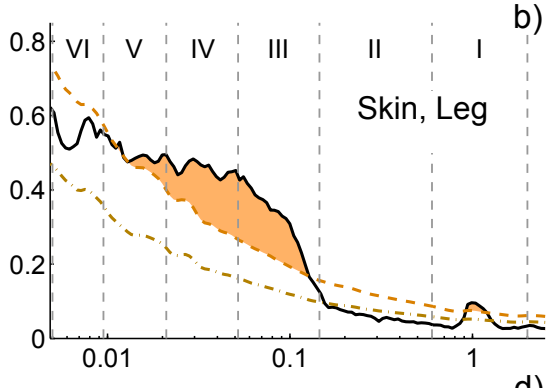

c)

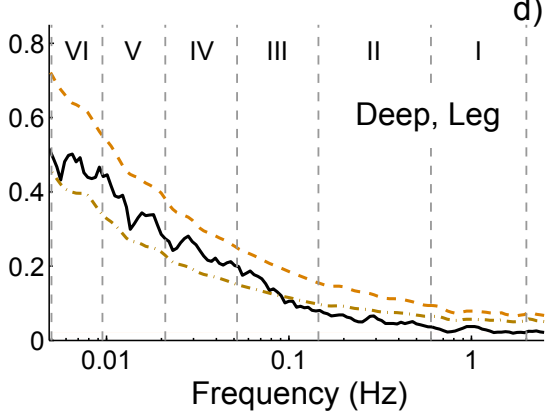

Fig. 4. Wavelet phase coherence between blood flow and oxygen saturation recorded from the arm $(\mathrm{a}, \mathrm{c})$ and the leg $(\mathrm{b}, \mathrm{d})$ and from two depths, skin $(\mathrm{a}, \mathrm{b})$ and deeper tissue $(\mathrm{c}, \mathrm{d})$. In each case, the median coherence for 88 subjects is indicated by the full line and the dashed lines show the mean, and two standard deviations above the mean, respectively, for the coherence calculated from 100 surrogate signals per subject. The coherence is significant across a wide frequency intervals for signals recorded from the skin $(a, b)$, but not so from the deeper tissue $(c, d)$.

frequencies for the arm compared to the leg (Fig. 3a). The specific effect of ageing on the parameters discussed in this paper will be presented elsewhere.

There is no coherence between blood flow and $\mathrm{Hb}$ oxygen saturation at the deeper recording, neither for the arm nor for the leg. We assume that this is due to the very different power spectra of oxygen saturation in the deeper tissue compared to the skin, together with the observation that the corresponding blood flow spectra are relatively similar. Whether this has a physiological explanation, or is related to the measurement technique, is beyond the scope of this study and will have to be further investigated.

Cross-spectra are commonly used to investigate relationships between signals by identifying frequency regions with high common power. Using wavelets, this can be further extended to determine these regions in the time-frequency plane [19]. However, common power at a particular frequency and time does not necessarily imply common cause. On the other hand, a consistent phase relationship is much more suggestive of causality between signals [20]. In the current study, the wavelet transform with logarithmic frequency resolution was used to extract phase information from the signals, and wavelet phase coherence was applied to identify oscillations 
A. Bernjak et al.

with consistent phase differences. This approach allowed us to find significant coherence even when the common power was low, which is important in the case of cardiovascular signals where low-frequency components make important, but not necessarily large, contributions. To determine instantaneous phases of oscillations, the wavelet transform applies the same approach as the Hilbert transform. The difference is that, with wavelets, the window is localized and slides within the signal, thus enabling one to trace the variations of phase in time.

Spectroscopic methods, such as used in NIRS or the O2C device, determine oxygen saturation in the capillary-venous part of the microcirculation. Thus the amount of oxygen bound to haemoglobin is assessed after distribution of oxygen into the tissue and the oxygen saturation signal therefore illuminates local oxygen utilization. Oscillations can be identified in the time series but, due to the complex nature of the blood flow in the capillaries, oxygen saturation on its own is insufficient to explain their origin. It is the $\mathrm{O} 2 \mathrm{C}$ facility for simultaneous measurements of flow and saturation in the same location that allows an analysis of their interactions to reveal further information about the mechanisms of oxygen consumption within the microcirculation. Wavelet phase coherence has identified a matching of phase differences over a wide frequency interval, clearly implying causal relationships between oscillations in blood flow and in oxygen saturation.

\section{Conclusion}

In conclusion, we have demonstrated for the first time the existence of coherence between simultaneously measured blood flow and oxygen saturation in the skin microcirculation of healthy subjects; no such coherence was observed in deeper layers containing skeletal muscle and larger vessels. The dynamical pattern of blood flow differs between these two layers but is scaled so that the normalised power of each oscillatory component is comparable. The dynamics of blood oxygenation is completely different between the two layers. While the power of the skin oxygenation signal comes mainly from low frequency components, that in deeper tissue comes mainly from oscillations at around the respiratory frequency. This is a novel and interesting result, and its physiological meaning has yet to be determined. It may be useful to establish the pattern of coherence in cardiovascular diseases and e.g. skin cancer.

\section{Acknowledgements}

We thank the subjects who volunteered for and participated in this research. The research was supported by the ESRC, UK 'New Dynamics of Ageing' programme. 


\section{References}

[1] A. Stefanovska, M. Bračič and H. D. Kvernmo, "Wavelet analysis of oscillations in the peripheral blood circulation measured by laser Doppler technique", IEEE Trans. Bio. Med. Eng. 46 (1999) 1230-1239.

[2] A. Stefanovska and M. Bračič, "Physics of the human cardiovascular system", Contemp. Phys. 40 (1999) 31-55.

[3] P. Kvandal, S. A. Landsverk, A. Bernjak, A. Stefanovska, H. D. Kvernmo and K. A. Kirkebøen, "Low frequency oscillations of the laser Doppler perfusion signal in human skin", Microvasc. Res. 72 (2006) 120-127.

[4] Y. Shiogai, A. Stefanovska and P. V. E. McClintock, "Nonlinear dynamics of cardiovascular ageing", Phys. Rep. 488 (2010) 51-110.

[5] Z. Y. Li, Y. H. Wang, Y. Li, Y. Wang, J. P. Li and L. L. Zhang, "Wavelet analysis of cerebral oxygenation signal measured by near infrared spectroscopy in subjects with cerebral infarction", Microvasc. Res. 80 (2010) 142-147.

[6] H. Obrig, M. Neufang, R. Wenzel, M. Kohl, J. Steinbrink, K. Einhaupl and A. Villringer, "Spontaneous low frequency oscillations of cerebral hemodynamics and metabolism in human adults", Neuroimage 12 (2000) 623-639.

[7] M. D. Stern, "In vivo observation of microcirculation by coherent light scattering", Nature 254 (1975) 56-58.

[8] G. E. Nilsson, T. Tenland and P. L. Öberg, "Evaluation of a laser Doppler flowmeter for measurement of tissue blood flow", IEEE Trans. Biomed. Eng. 27 (1980) 597-604.

[9] D. M. Mancini, L. Bolinger, H. Li, K. Kendrick, B. Chance and J. R. Wilson, "Validation of near-infrared spectroscopy in humans", J. Appl. Physiol. 77 (1994) 2740-2747.

[10] R. Boushel and C. A. Piantadosi, "Near-infrared spectroscopy for monitoring muscle oxygenation", Acta Physiol. Scandinav. 168 (2000) 615-622.

[11] A. Villringer and B. Chance, "Non-invasive optical spectroscopy and imaging of human brain function", Trends. Neurosci. 20 (1997) 435-442.

[12] C. E. Thorn, H. Kyte, D. W. Slaff and A. C. Shore, "An association between vasomotion and oxygen extraction", Am. J. Physiol-Heart C. 301 (2011) H442-H449.

[13] A. Stefanovska, "Dynamics of blood oxygenation gives better insight into tissue hypoxia than averaged values", Am. J. Physiol-Heart C. 296 (2009) H1224-H1226.

[14] A. Bandrivskyy, A. Bernjak, P. V. E. McClintock and A. Stefanovska, "Wavelet phase coherence analysis: Application to skin temperature and blood flow", Cardiovascular Engineering 4 (2004) 89-93.

[15] L. W. Sheppard, V. Vuksanović, P. V. E. McClintock and A. Stefanovska, "Oscillatory dynamics of vasoconstriction and vasodilation identified by time-localized phase coherence", Phys. Med. Biol. 56 (2011) 3583-3601.

[16] T. Schreiber and A. Schmitz, "Improved surrogate data for nonlinearity tests", Phys. Rev. Lett. 77 (1996) 635-638.

[17] A. G. Tsai and M. Intaglietta, "Evidence of flowmotion induced changes in local tissue oxygenation", Int. J. Microcirc. Clin. Exp. 12 (1993) 75-88.

[18] D. Goldman and A. S. Popel, "A computational study of the effect of vasomotion on oxygen transport from capillary networks", J. Theor. Biol. 209 (2001) 189-199.

[19] C. Torrence and G. P. Compo, "A practical guide to wavelet analysis", Bull. Am. Meteorol. Soc. 79 (1998) 61-78.

[20] A. Grinsted, J. C. Moore and S. Jevrejeva, "Application of the cross wavelet transform and wavelet coherence to geophysical time series", Nonlin. Process Geophys. 11 (2004) 561-566. 